\title{
Initial Implementation and Operational Use of TASAR in Alaska Airlines Flight Operations
}

\author{
David J. Wing ${ }^{1}$ \\ Kelly A. Burke, Ph.D. ${ }^{2}$ \\ NASA Langley Research Center, Hampton, VA 23681, USA \\ Jeffrey Henderson, Ph.D. ${ }^{3}$ \\ Engility Corporation, Andover, MA 01810, USA \\ Robert A. Vivona ${ }^{4}$ \\ Aurora Flight Sciences, Cambridge, MA 02142, USA \\ Jared Woodward ${ }^{5}$ \\ Alaska Airlines, Seattle, WA 98188, USA
}

\begin{abstract}
NASA has been developing and testing the Traffic Aware Strategic Aircrew Requests (TASAR) concept for aircraft operations featuring a NASA-developed cockpit automation tool, the Traffic Aware Planner (TAP), which computes route changes compatible with nearby traffic and airspace constraints to improve flight efficiency. The TAP technology is anticipated to save fuel, flight time, and operating costs and thereby provide immediate and pervasive benefits to the aircraft operator. Alaska Airlines is partnered with NASA to implement and evaluate TASAR in revenue service. This paper will describe activities undertaken to achieve TASAR operational status at Alaska Airlines, and it will present preliminary results from initial flight operations.
\end{abstract}

\section{Introduction}

A

dvancements in airspace operations and aircraft technology are typically born as high-level concepts developed in research laboratories and then matured through years of analyses, simulations, and flight testing. The ultimate success of these new operations and technologies, though, are determined only after they achieve operational use. Transitioning a research concept from laboratory implementation to operational deployment and use by an airline can be a complex undertaking that takes many years and significant planning and multi-organizational coordination to achieve. A partnership between the National Aeronautics and Space Administration (NASA) and Alaska Airlines (Alaska) has achieved such an undertaking through the implementation of Traffic Aware Strategic Aircrew Requests (TASAR) onboard three designated Boeing 737-900ER aircraft [1]. On Sept 26, 2017, Alaska conducted the first commercial revenue flight with NASA's TASAR software, Traffic Aware Planner (TAP), operating onboard. Reached in just two years, this milestone marks the achievement of transitioning TASAR, a NASA concept for en route flight path optimization, into airline operational use and evaluation. Assisting NASA and Alaska with this achievement were industry leaders Gogo Commercial Aviation (Gogo), United Technologies Corporation Aerospace Systems (UTAS), and Aviation Communications \& Surveillance Systems (ACSS), as well as NASA contractors. Specifically, subcontractor Engility Corporation was central in developing the TAP software for NASA and adapting it to Alaska's aircraft for the TASAR operational evaluation.

\footnotetext{
${ }^{1}$ ATM Research Engineer, Crew Systems \& Aviation Operations, Mail Stop 152, AIAA Associate Fellow

${ }^{2}$ Human Factors Research Scientist, Crew Systems \& Aviation Operations, Mail Stop 152, AIAA Member

${ }^{3}$ Senior Research Engineer, ATRE Department, 2 Tech Dr., AIAA Senior Member

${ }^{4}$ Lead Autonomy Engineer, 90 Broadway, Suite 11, AIAA Associate Fellow

${ }^{5}$ Technical Pilot, Alaska Airlines Flight Operations, 2651 S $1192^{\text {nd }}$ St.
} 
TASAR leverages the emerging revolution of the "connected aircraft" where access to operational information by systems onboard and off the aircraft becomes ubiquitous [2]. Technologies such as Electronic Flight Bags (EFB), Aircraft Interface Devices (AID), and Airborne Control Processing Units (ACPU) offer computing power and unprecedented Internet-Protocol-based connectivity to EFB software applications. Together, they are changing the landscape in which new operational capabilities are envisioned and implemented. As illustrated in Figure 1, NASA's TASAR concept [1] features a route optimization engine that consumes data drawn from a variety of sources:

1. The onboard flight management system (e.g., aircraft state and route data),

2. Other onboard avionics systems (e.g., traffic data, weather radar data), and

3. Ground-based data sources (e.g., wind forecasts, convective and turbulence weather products, special use airspace (SUA) activation schedules).

Combined with pilot inputs via the TAP human-machine interface (HMI), these data are processed by a powerful search algorithm that identifies opportunities to request changes in the aircraft's planned lateral path and/or altitude from air traffic control (ATC). TASAR's goal is to re-optimize the flight using the latest conditions to achieve a specified objective, such as minimizing fuel burn, flight time, or overall trip cost. By taking into account ATC factors of concern such as traffic proximity, SUA status, and weather, TASAR requests for rerouting should be more easily coordinated with dispatch (for airline operators) and have an increased likelihood of ATC approval, thereby increasing the realized benefits to the operator. The TAP software is NASA's state-of-the-art prototype EFB application that achieves the TASAR concept and was awarded NASA Software of the Year in 2016 [3].

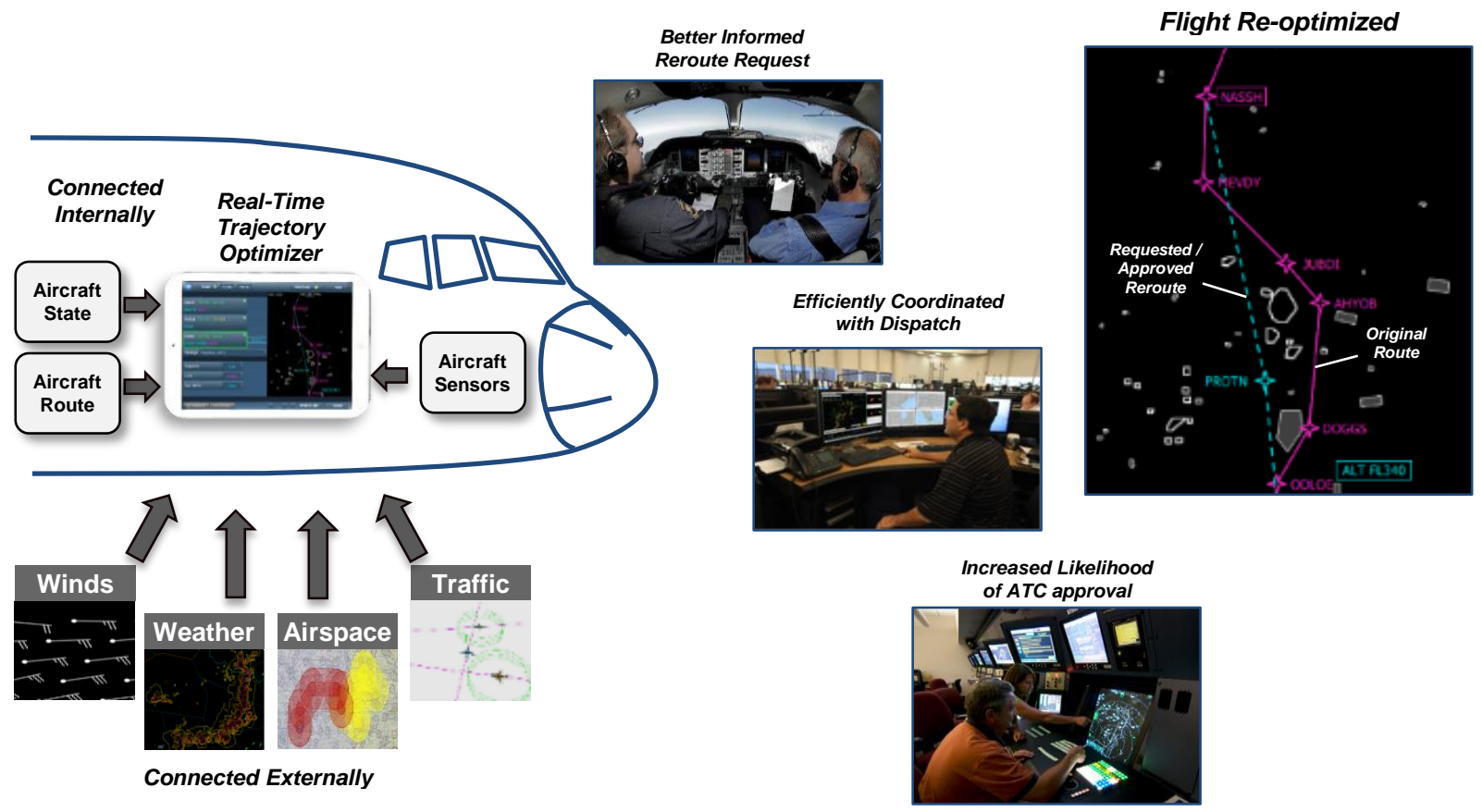

Figure 1. TASAR leverages data connectivity and a real-time trajectory optimizer to produce optimized flight benefits.

Previous publications have described TASAR's research and development history [4] [5], benefits analyses [6] [7] [8], safety and certification analyses [9] [10], software development [3] [11], and flight testing [12]. The current paper focuses specifically on the activities undertaken to implement TASAR at Alaska Airlines as a fully functioning EFB application approved by the Federal Aviation Administration (FAA) for operational use in revenue service. Section II will describe the multi-disciplinary, multi-organizational collaboration leading to the Alaska Airlines implementation. Section III will describe the multi-stage implementation plan leading to full operational use and discuss the various tests and analyses performed during those stages. Section IV will present conclusions. 


\section{Multi-Disciplinary Multi-Organizational Collaboration}

Achieving the first operational TASAR flight on Alaska Airlines aircraft required an extensive, multi-disciplinary collaboration. Organizations and individuals contributing to this project included:

- NASA research, legal, licensing, software release, and airworthiness review offices;

- NASA-contracted software developers, testers, and analysts;

- Industry hardware, software, and certification specialists;

- Government regulatory organizations; and

- Airline departments including flight operations, avionics engineering, aircraft maintenance, cabin systems engineering, mobile technology, crew training, regulatory compliance management, and flight dispatch.

The extensive coordination required to ensure all of these disciplines were adequately engaged and had their requirements met (in the proper sequence) highlights the complexity of the otherwise simply stated task of installing research software on a commercial aircraft. With little to no ability to modify software or hardware after installation, all elements of the project required careful planning. The contributions of these various entities, though crosscutting throughout the project, aligned primarily with the following six threads of activity.

\section{A. Project Management}

The TASAR operational evaluation was formulated as a collaborative activity between NASA and Alaska Airlines and was formalized in a 2015 Space Act Agreement between the two organizations. ${ }^{6}$ The NASA sponsors within the Airspace Operations and Safety Program were initially the Concepts and Technology Development Project (CTD) and subsequently the Airspace Technology Demonstration (ATD) project (third sub-project, ATD-3). CTD developed mid Technology Readiness Level (TRL) concepts and technologies for airspace operations, and ATD demonstrates high TRL concepts and technologies in operationally relevant environments leading potentially to technology transfer. The TASAR collaboration grew from Alaska's participation as an evaluator in the 2013 TASAR flight trial [12] in which the Director of Fleet Technology personally evaluated the technology in flight and confirmed its alignment with Alaska's strategic technology vision.

The Space Act Agreement defined the basic objectives and responsibilities of the collaboration:

1. NASA to adapt TAP software for compatibility with Alaska trial aircraft;

2. Alaska to install EFB hardware (with all necessary data connections) and TAP software;

3. Alaska to acquire FAA operational approval;

4. Alaska to conduct evaluation flights in revenue service (anticipated up to one year of flights);

5. NASA to analyze data for operational benefits;

6. NASA to update TAP software based on interim findings.

To achieve these objectives, NASA and Alaska assembled the multi-organizational team shown in Figure 2. With leadership jointly provided by NASA and Alaska TASAR project leads, the team pooled together a diverse set of expertise. NASA researchers and contractors contributed expertise in the TAP software code and its capabilities, including adapting it to new aircraft environments, software testing, and data analysis to confirm proper operation and realized benefits. Alaska Avionics Engineering contributed expertise in hardware integration, software installation, and avionics regulatory compliance management. NASA supporting organizations contributed expertise in systems engineering, legal agreements, software release processes, and Agency-required reviews and approvals (Institutional Review Board, Airworthiness and Safety Review Board, etc.). Organizations internal to Alaska Airlines contributed expertise in flight dispatch, mobile information technology (IT), flight operations regulatory compliance management, aircraft performance and fuel efficiency, and crew training,. Organizations external to Alaska Airlines,

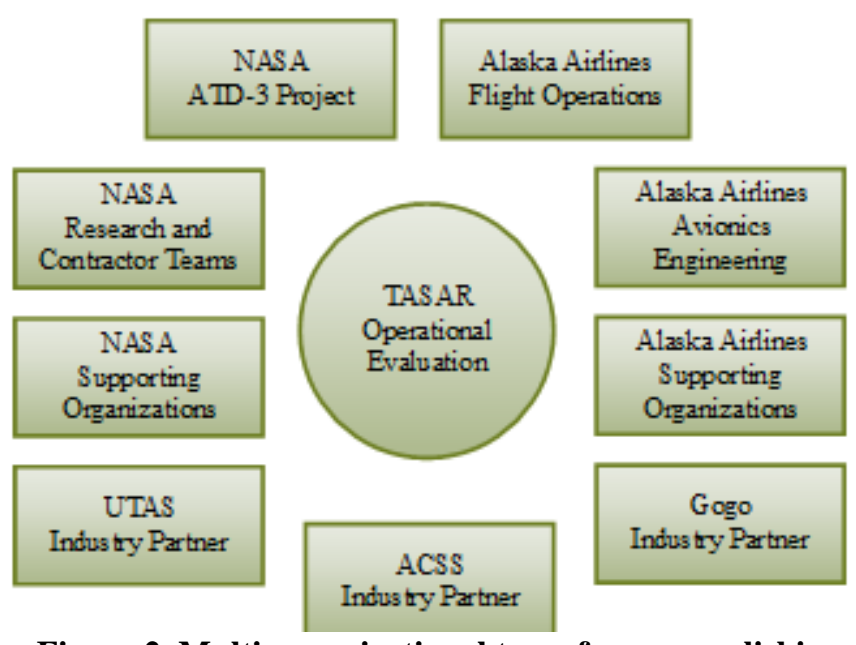

Figure 2. Multi-organizational team for accomplishing the TASAR operational evaluation.

\footnotetext{
${ }^{6}$ The NASA-Alaska Airlines TASAR partnership kickoff meeting was held on September 22, 2015.
} 
namely UTAS, Gogo, and ACSS, each contributed expertise in their hardware platforms, software integration and testing, software quality assurance and packaging, IT integration and security, and aircraft and technology certification. Each of these industry partners played a crucial role in the success of the project, working cooperatively with each other and the NASA/Alaska teams through weekly meetings and periodic integration testing in laboratories and onboard the aircraft.

\section{B. Hardware Installation and Certification}

TAP is intended to be installed on existing EFB hardware systems and is designed to support a variety of potential architectures of installed and portable EFB hardware. Alaska selected a multi-component Class 2 EFB architecture to host the TAP software, consisting of two iPads ${ }^{\circledR}$, two Tablet Interface Modules (TIM ${ }^{\circledR}$ ), an AID, and an ACPU. This configuration enables two independent instances of TAP to be installed on separate hardware platforms for project risk reduction and to enable the option of the Captain and First Officer to independently have access to TAP for evaluation. The TASAR hardware/software architecture for Alaska's trial aircraft is shown in Figure 3.

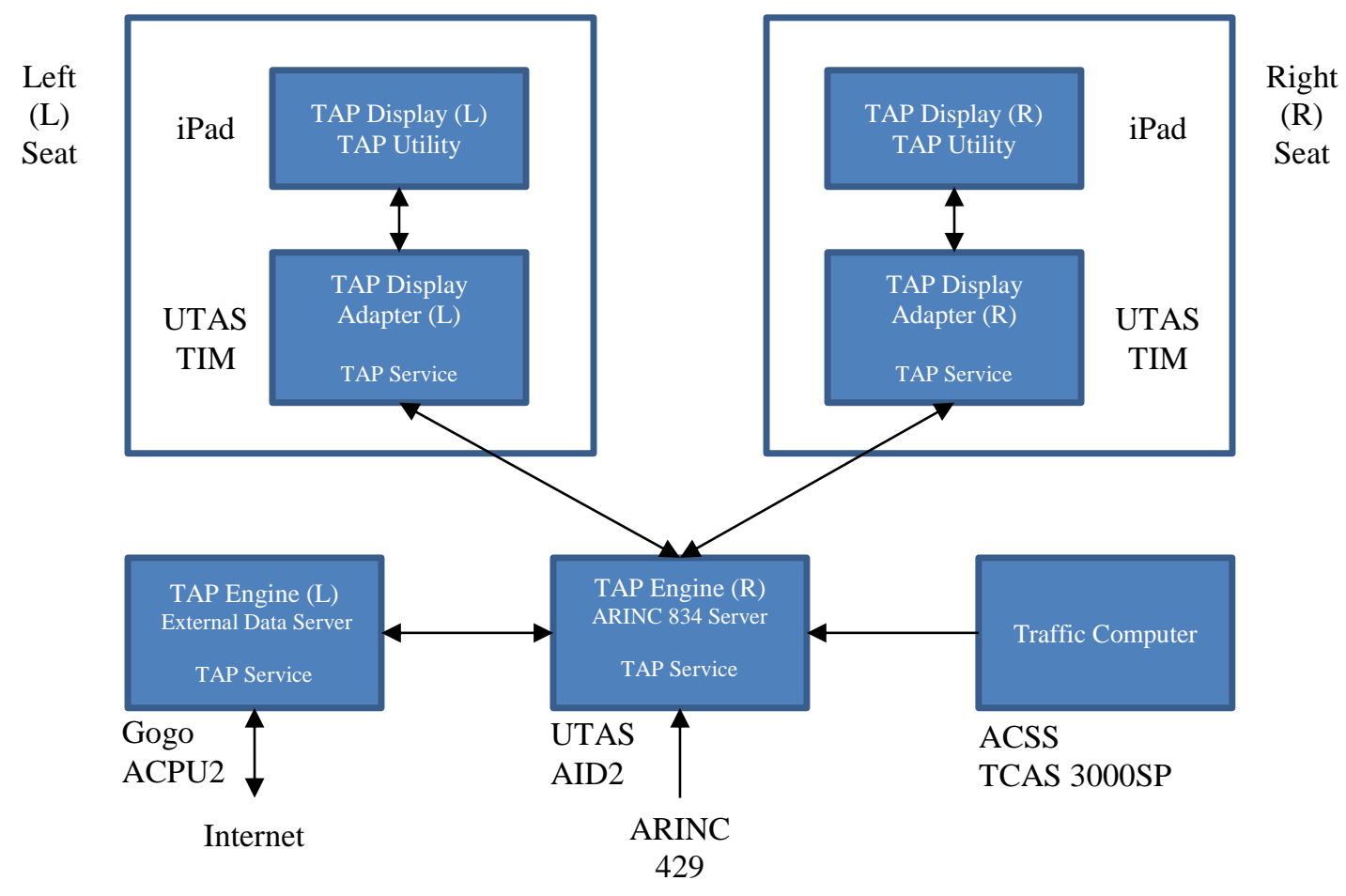

Figure 3. TASAR architecture on Alaska Airlines trial aircraft. $L$ and $R$ refer to system components supporting left (Captain) and right (First Officer) instantiations of the TAP software.

Pre-installed Gogo ACPU hardware was leveraged for this project, with internal firmware adaptations made by Gogo to host TAP software and provide first-time (for Alaska) internet connectivity to the cockpit. These adaptations were timed to coincide with a pre-planned ACPU firmware update cycle. UTAS AID and TIM hardware were newly installed for this project and were adapted by UTAS to host TAP software and provide first-time (for Alaska) avionics connectivity (via the ARINC 429 data transfer standard for aircraft avionics) to the iPad EFB. This installation was authorized via a Supplemental Type Certificate (STC) - a formal approval process between UTAS and the FAA to recertify the aircraft type with the AID and TIM hardware installed. ${ }^{7}$ Subsequently, a separate STC was acquired to connect UTAS and Gogo hardware in flight, which is a requirement to ensure security issues associated with connecting the internet/passenger domains to the cockpit are addressed. ${ }^{8}$ Pre-installed ACSS Traffic Alert and Collision Avoidance System (TCAS) hardware was leveraged to provide traffic data to TAP via Automatic Dependent Surveillance Broadcast In (ADS-B In). Modifications included TCAS firmware and wiring adaptations made by

${ }^{7}$ AID-TIM installation STC was achieved on March 6, 2017

${ }^{8}$ AID-ACPU connectivity STC was achieved on August 25, 2017 
ACSS to feed the TCAS 3000SP data into the EFB system via the AID, which resulted in a third STC for this project, accomplished by ACSS, as well as a Technical Standard Order for the modified TCAS hardware. ${ }^{9}$

All STC efforts involved tight coordination with Alaska Avionics Engineering and Alaska's Compliance Management Office. Where tests onboard an aircraft were required as part of the STC efforts, the principal Alaska Avionics Engineer scheduled one of the three TASAR aircraft for the test and then subsequently activated the systems once the STC was finalized. The upgrades were then applied to the remaining two aircraft.

In addition to STCs to install the hardware and connections, operational approval is also required from the FAA for use of the EFB and the software application during flight operations. Alaska achieved two operational approvals from the FAA via an update to their Operations Specification A061, one to operate the Class $2 \mathrm{EFB}^{10}$ itself and another to install and use the TAP software as a Type B application. ${ }^{11}$

\section{Software Adaptation and Deployment to Alaska Airlines}

The highly regulated aircraft environment added an additional challenge: conforming to industry-formalized practices for software verification, packaging, and deployment/installation. Overseen by Alaska Airlines Avionics Engineering and performed by Gogo and UTAS, these practices required the complete lock-down of TAP software by NASA and Engility several months in advance of each installation. The entire team integrated their efforts to make this process a success. Figure 4 shows the TAP software application displayed on the Alaska Airlines iPad EFB in a full-motion flight simulator.

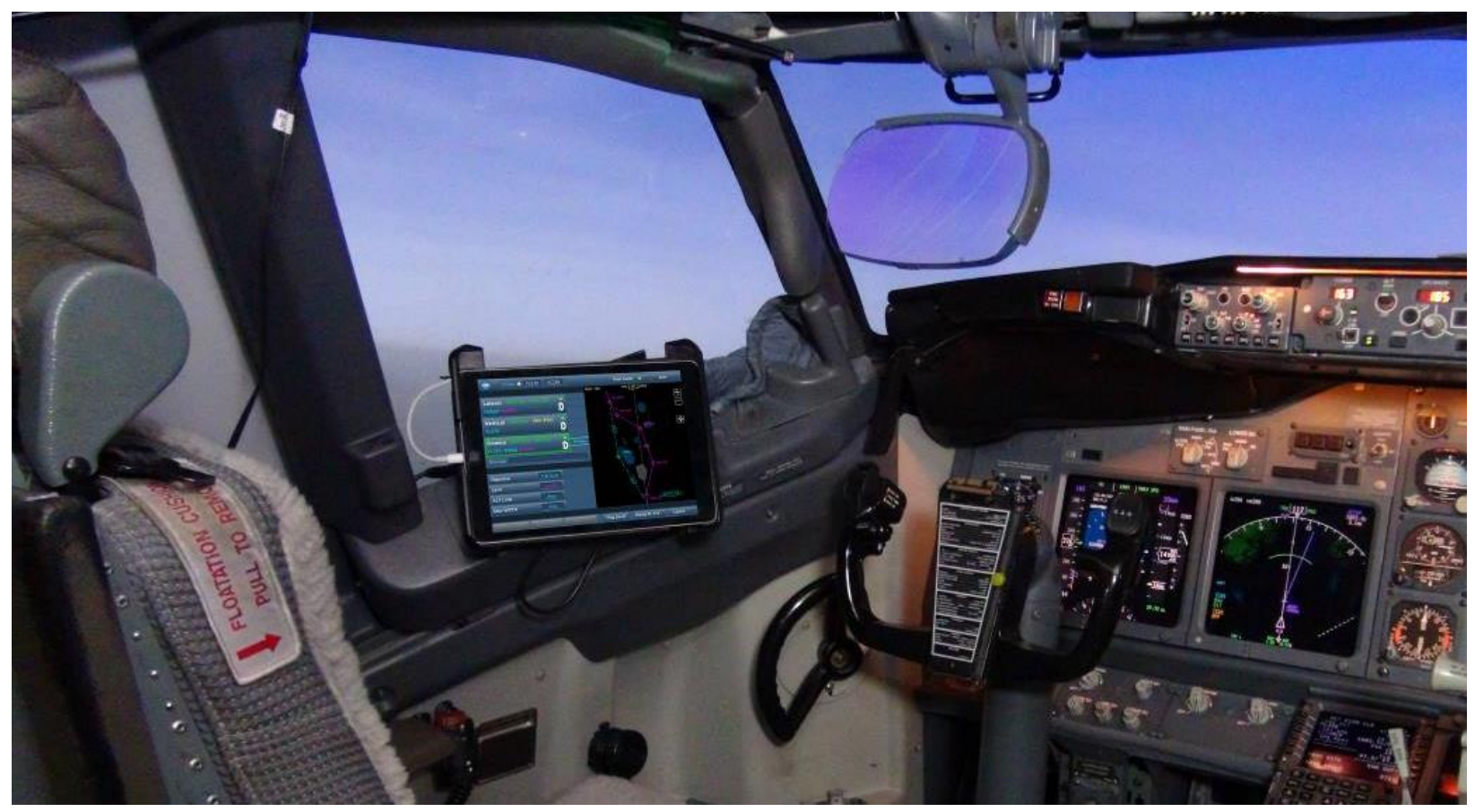

Figure 4. TAP software application displayed on the Alaska Airlines iPad EFB in a full-motion flight simulator. Photo credit: Janet Ann, Alaska Airlines.

Deploying TAP to work on a particular aircraft requires several things to be addressed: ensuring the hosting hardware can support the TAP application (e.g., operating system, processor speed, memory footprint, partitioning), accessing the real time data TAP consumes from onboard avionics and internet data sources, and installing aircraft performance data from which TAP can model the aircraft behavior for accurate trajectory prediction. Since none of these things are standard for all aircraft, each installation of TAP in a new aircraft environment requires some level of adaptation to that environment and/or by that environment. In the Alaska deployment, Gogo, UTAS, and Engility worked together to adapt TAP and the hosting hardware (i.e., ACPU, AID, and TIM) for mutual compatibility, enabling TAP to compile, run, support inter-process communications, and be protected on the hardware. The industry

${ }^{9}$ AID-TCAS connectivity STC was achieved on March 26, 2018

${ }^{10}$ Class 2 EFB operational approval was achieved on June 20, 2017

${ }^{11}$ TAP software operational approval was achieved on August 7, 2017 
alternate data source for ADC parameters had further problematic implications discovered during the initial flight trials, which are discussed in Section III.

\section{Aircraft Performance Modeling}

The TAP software includes a trajectory generation (TG) algorithm [13] that predicts future 4D positions and fuel states along the FMS active route and TAP-generated candidate reroutes. The versatile TAP TG has been demonstrated to support aircraft models of different types (in particular, turbojet vs. turboprop aircraft) using aircraft performance models (APM) at different levels of detail and in different formats. For the Alaska implementation, the expectation was for TAP to use the APM dataset provided by the Original Equipment Manufacturer (OEM), in this case Boeing, using the industry "Standardized Computerized Airplane Performance" (SCAP) format [14]. Unfortunately, it was not possible to proceed on this path for two reasons. First, the SCAP standard is used by OEMs primarily for takeoff and landing data, and en route cruise performance modeling has not kept up with this standard in the industry. Second, the APM data usage agreement between NASA and Boeing was not completed in time for the project, though it was eventually executed. An alternative APM approach was therefore pursued and implemented using the Eurocontrol "Base of Aircraft Data Version 4" (BADA 4) aircraft performance modeling specification and dataset [15]. BADA 4 enables modeling aircraft behavior with increased levels of precision in aircraft performance parameters over the entire flight envelope. A NASA agreement with Eurocontrol, with concurrence received from the OEM, authorized NASA to implement the BADA 4 specification in TAP and deploy it with the Boeing 737900ER BADA 4 dataset to Alaska Airlines for the TASAR Operational Evaluation. This deployment is the first approved use of BADA 4 in an airborne application.

One element lacking from the BADA 4 APM was a specification of economy cruise speeds, or ECON speeds (i.e., the FMS Mach/CAS schedule when the pilot has selected the ECON setting for FMS speed management). Airborne applications like TAP are dependent on accurate speed modeling for the particular aircraft, matching speeds as controlled by the aircraft's FMC. To compensate for the missing ECON speed data, an Alaska Airlines flight simulator was used to derive a speed table including dependencies on appropriate flight parameters. TAP's TG was modified to read the speed table as required. This limitation of BADA 4 was communicated back to the Eurocontrol BADA team to help improve the model going forward.

\section{Crew Training}

To obtain FAA approval for software to be used in the cockpit, pilots must receive training prior to using it during operational flights. In an effort to enable near-term deployment of TAP software on commercial revenue flights with Alaska and to reduce time and resources potentially required by Alaska to develop TAP training, NASA developed the TAP Training Package, which includes the TAP Computer Based Training Module, the TAP Operations Procedures Manual, and the TAP Flight Manual Bulletin. This section describes the development and evaluation process of this training package.

Computer based training (CBT) is the use of computers to provide an interactive, instructional experience in which the computer is the primary mode of instruction. CBT modules typically contain material of a specific field, are interactive, self-directed, and self-paced, and often include hypertext, multimedia elements, texts, graphics, animation, sound, and interaction with a simulated version of the actual software on which the user is being trained. Using the graphic visualizations of actual software and materials as well as an interactive combination of different media units, a CBT is an effective form to acquire knowledge. NASA chose to develop a CBT module as the main TAP training approach because CBT is the primary methodology currently used by airlines for crew training (i.e., type rating, operations, and organizational requirements) and has been shown to be an effective and convenient method for training pilots. Throughout the development process, NASA collaborated closely with Alaska Crew Training, Mobile IT, and Flight Operations to ensure that the CBT was developed using software compatible with their Learning Management System (LMS) and would further achieve compatibility with their training requirements.

As depicted in Figure 6Error! Reference source not found., the design and development process leveraged a user-centered, iterative, design approach where each phase of the development cycle included testing with pilots. The TAP CBT was used to train pilots during several activities where the pilots used the TAP software, including a TASAR human-in-the-loop simulation experiment and a TASAR flight trial. Several metrics were used to determine the extent to which the TAP CBT was an effective method for training pilots on the operation and use of the TAP software, including subjective (Training Effectiveness Questionnaire) and objective (Training Criterion Checklist) measures. The results of the data analyses were implemented in the next iteration of the CBT (i.e., TAP CBT Generations $2 \&$ 3). Updates included: reducing the length of the CBT (from a 55-minute average run time to 30 minutes) to accommodate airline time constraints; adding a professional voice over; incorporating a tablet-style interface to accommodate administration on the iPad EFB; adding the ability to navigate through modules (pause, replay, etc.); 


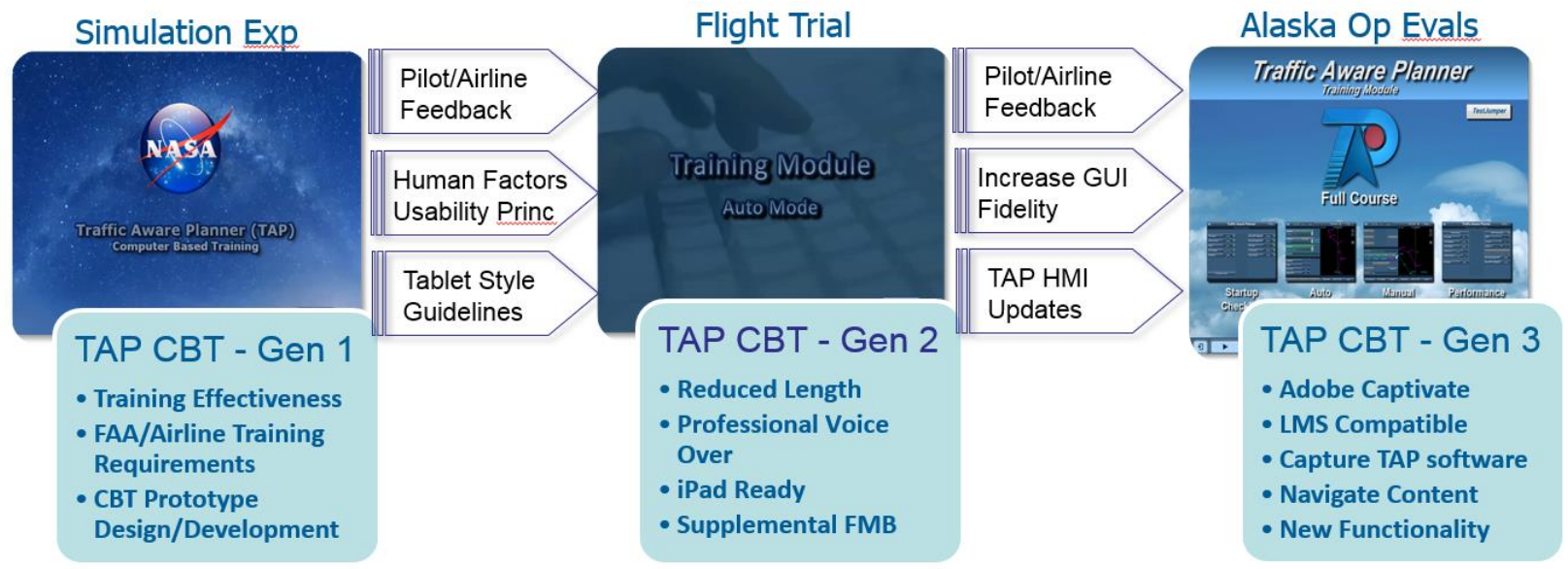

Figure 6. TAP CBT development and testing process.

and increasing the fidelity of the CBT by using the actual TAP software in its creation and a realistic flight scenario. The result of this user centered iterative design process was a state-of-the-art TAP CBT Module (see Fig. 7) which effectively trains pilots on TAP use, operation, and procedures.

While the TAP CBT was designed to serve as the primary means of training pilots on "how to use" TAP, supplemental training materials were developed to enhance the training experience. The TAP Flight Manual Bulletin was designed to complement the TAP CBT by providing additional "under the hood" details about the TAP software and describing TAP features and functions thoroughly. This document provides pilots with a solid foundation to the purpose of the TAP software, how it calculates trajectory optimizations, and how the pilot can operate the various functions to more effectively use TAP. This document also prepares the pilot for the TAP CBT and enhances the learning experience and knowledge retention. The TAP Operating Procedures Manual is also included in the TAP Training Package and was designed to provide pilots with a comprehensive stand-alone reference after the pilot receives TAP training. Both of these documents will be pushed through the LMS to the pilots iPads so they may reference them at any time.
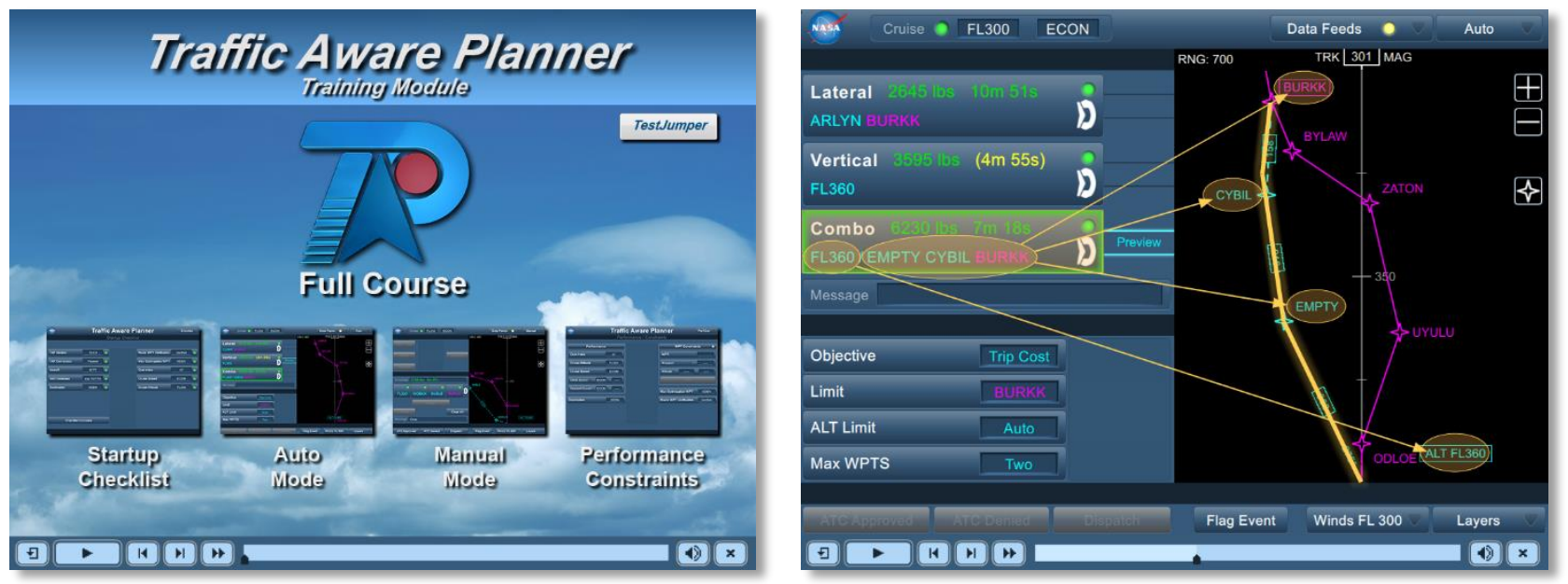

Figure 7. The TAP Computer Based Training Module.

\section{E. Usability Assessment.}

During the Alaska Operation Evaluation, NASA will collect usability data from the pilots as they use the TAP software during revenue flights. This will enable NASA to identify design considerations that can be improved upon in future TAP builds. Also, it will enable communication between Alaska end users and the research and development team allowing for a more effective implementation of the software.

A three-step plan is in place to collect data from the pilots in the least intrusive and time consuming manner. First, we will ask the pilots to respond to a brief electronic questionnaire accessible on the EFB either during or immediately 
after the flight in which they interacted with the TAP software. The purpose of this questionnaire is to elicit information from the pilots about usability and functionality concerns which may have occurred during the flight. It is important to try to capture this information while it is fresh in the pilot's mind. For example, the TAP HMI has a 'Flag' button for the pilots to press anytime they encounter an issue they would like to bring to the attention of the research team. We will ask the crew if they used this button during the flight and to briefly explain the issue they would like us to be aware of. This will allow the research team to correlate the data tag in the log with the explanation provided by the pilot.

The second means for collecting usability data will be via the Slack ${ }^{\circledR}$ application. This application is currently used by Alaska pilots and enables a team to easily communicate and share information. NASA coordinated with Alaska Mobile IT to develop a TAP thread which will include Alaska pilots and NASA researchers. Slack will allow for the NASA research team and the pilots to easily communicate about the use of TAP, share information about their experience interacting with the software, ask questions, discuss concerns, etc. The pilots can also easily capture and send screen shots of the display to the researchers to further communicate usability issues. This method of communication will enhance the implementation experience for both the end users and NASA.

Finally, a more comprehensive questionnaire regarding TAP usability and acceptability will be administered intermittently during the operational evaluation. The questionnaire will enable the research team to continue to monitor the end user experience with the TAP software across time as flight crews become more experienced using it during revenue flights.

\section{F. TAP Data Retrieval for Analysis}

The previous sections described the activities required to get TAP "off the ground" for operational evaluation, including installing the required hardware and connectivity (with required certifications and operational approvals); adapting and deploying the TAP software to the particular aircraft; training the flight crews on effective TAP use, operations, and procedures in flight; and preparing usability assessments. Equally important to the operational evaluation, however, was the ability to retrieve data electronically recorded by the TAP software itself during the flights. Depending on the type of data analysis to be performed, TAP is capable of recording a smaller or larger amount of data. It can record just enough data for analyzing achieved benefits (e.g., actual flight time and fuel burn saved as a result of TAP's usage by the crew), or if needed, it can record a much more extensive "debug" level of data enabling analysts to effectively replay the entire flight through TAP "playback" mechanisms and identify potential software problems. Though it would be possible to downlink these datasets during the flight, it is cost-ineffective due to the volume of data recorded by TAP. Therefore a method was developed to automatically retrieve the data files after landing when access to terrestrial modems were available. (Manual retrieval would again be cost prohibitive, considering the potential frequency of TASAR flights.) Multiple options for offloading the data files were developed. Prior to shutting down for a flight, TAP could automatically collect the files from each hardware unit and bundle them in one onboard central location for ease in offloading. Alternatively, to support distributed offloading, the TAP file naming structure was developed such that separately offloaded files could later be reunited, including distinguishing files from the Captain's and First Officer's TAPs. UTAS, Gogo, and NASA each developed scripts that together automated the entire data retrieval process after each flight, with only limited manual steps performed where required by Alaska Airlines for data transmission to NASA and Engility for analysis.

\section{Multi-Stage Implementation Plan}

The long lead times required for hardware certification and software lock-down made it imperative to verify the Alaska TASAR system as early as possible to detect and resolve any faults with minimum delay to the project. To this end, the NASA/Engility team devised a multi-stage implementation plan to reduce project risk for schedule and cost. The objective of the multi-stage plan was to verify required data flows, software performance, and operational readiness through a series of on-aircraft ground and airborne tests. The challenge was to maximize on-aircraft testing appropriate to the evolving certification/approval phase, while minimizing operational costs to Alaska Airlines (e.g., aircraft downtime, crew training). The plan consisted of four testing "stages" (numbered Stage 0 through 3, where Stage 0 was targeted for ground testing and the remaining for airborne testing).

\section{A. Stage Definitions}

The objective of Stage 0 was to verify that each of TAP's required input parameters (e.g., present position latitude) mapped correctly to the appropriate avionics data source, that each data parameter was indeed received (with expected characteristics like data rate), and that TAP properly decoded each one. Given that the software team developed the mapping and decoding scheme based on documentation, the risk to be averted was any difference between the 
documentation and the installed hardware. The initial installation of the AID in the aircraft for certification testing presented the earliest opportunity to conduct this test. The test required power to be applied to all relevant avionics, the ARINC 834 server to be active with connections to the appropriate avionics, and identifiable data to be received on each parameter's port. As will be discussed in the upcoming section, most of the parameters could be static-tested on the ground without a fully running (or installed) version of TAP. Some parameters were only available in flight, and so a single airborne Stage 0 data collection was included. See Section B for Stage 0 analyses and sample results.

The objective of Stage 1 was to verify TAP was performing properly in flight prior to Alaska pilots actually using the software. At a high level, this "shadow mode" test, to be conducted automatically without a pilot's user interface (i.e., the TAP Display), was intended to confirm that TAP was processing the data it received from avionics and internet sources, computing reasonable reroute solutions suitable for operational use, and generating solutions resulting in accurate time/fuel savings. In practice, the Stage 1 test addressed specific sub-objectives: confirm the findings of Stage 0 with in-flight data; assess TAP's performance when running in the actual flight hardware environment; assess the stability characteristics of computed solutions; and measure the accuracy of TAP's time and fuel saving predictions. See Section C for Stage 1 analyses and sample results. As will be discussed, the certification and deployment schedule necessitated Stage 1 being subdivided into Stage 1a and 1b. At the time of this publication, Stage 1 was complete and Stage 2 flight operations were ready to start.

The objectives of Stage 2 and 3 are to acquire initial operational feedback from Alaska pilots actively using TAP and to assess achieved operational benefits from making TAP-inspired reroute requests to ATC. In Stage 2, a small group of evaluation pilots from Alaska Flight Operations will perform the initial assessment to establish standard operating procedures (SOP) for TAP usage and to identify any particular issues requiring attention before expanding the trial to more pilots. The larger "debug" level of data collection in this stage will enable in-depth analysis of TAP performance and post-flight review of particular events identified by the evaluation pilots in flight. If necessary, changes would be made to the software and/or SOPs to support a successful trial. Once this initial assessment is complete, Stage 3 will commence with a gradual expansion to a larger segment of Alaska pilots, including the implementation of a formal training program using materials developed by NASA. Data collection in Stage 3 will reduce to the smaller dataset suitable for ongoing analysis of achieved benefits across all TASAR flights throughout the operational evaluation.

\section{B. Stage 0 Analysis and Results}

During Stage 0 testing, Alaska Airlines and Engility technical staff ran TAP on a Windows ${ }^{\circledR}$ laptop computer, onboard the aircraft, connected to a UTAS TIM via an Ethernet-to-USB connection. A laptop was used to host TAP because Stage 0 preceded operational approval to install TAP on the UTAS AID and Gogo ACPU. ${ }^{13}$ Instead of processing the avionics data, TAP recorded STAP messages from the AID to a binary file. During data collection, a separate process monitored this binary file to confirm that all required Stage 0 data had been successfully received and decoded.

Stage 0 was executed in three parts: Stage 0 Hangar, Stage 0 Taxi, and Stage 0 Airborne. During Stage 0 Hangar, Alaska Airlines personnel used a ground-test apparatus to apply controlled signals to aircraft sensors to make certain avionics generate non-zero data as if the aircraft was airborne, a process that worked for most of the avionics data. However, the FMS requires the aircraft to be moving above 40 knots before complete active route waypoint information was generated. For this reason, Alaska Airlines pilots conducted a high-speed taxi on SeaTac (KSEA) runway $34 \mathrm{C}$, during which the route waypoint data was collected. The Stage 0 Taxi operation was made possible through coordination with FAA controllers in KSEA Tower.

Stage 0 data analysis thus far confirmed that TAP received the majority of expected input parameters. However, during the initial Stage 0 data collections, TAP did not receive some input parameters from the FMC (i.e., barocorrected altitude, CAS, altitude rate, route data, and guidance mode state). For this reason, the TASAR team added two additional Stage 0 Hangar data collection sessions that successfully captured the missing FMC data. A subsequent Stage 0 Airborne data collection leveraged a planned repositioning flight to collect guidance mode data corresponding to states that could not be engaged on the ground. ${ }^{14}$ Finally, once the ADS-B system was installed, another hangar data collection was used to collect and verify ADS-B data. ${ }^{15}$

After each data collection, an analysis was performed on inter-arrival times of TAP input parameters (e.g., present position latitude) received via the STAP data feed. Though there was no specific target, the inter-arrival times should generally be on the same order of magnitude as TAP's one-second processing time frame so that TAP-generated

\footnotetext{
${ }^{13}$ Stage 0 ground testing began on March 7, 2017.

${ }^{14}$ Stage 0 airborne testing occurred during an aircraft repositioning flight on May 5, 2017.

${ }^{15}$ Stage 0 testing of ADS-B data occurred on February 7, 2018.
} 
reroutes are based on up-to-date data. Successfully applied during previous TASAR flight trials, this analysis was reapplied to the Alaska Airlines Boeing 737-900ER since its avionics are different from the TASAR flight trial aircraft and were expected to generate data with different characteristics.

Inter-arrival times corresponding to present position latitude, universal time coordinated (UTC), and the complete active route during the Alaska Airlines repositioning flight are shown in Table 1 as a sample. The table shows that TAP received 12,910 latitude updates at an average rate of 0.21 seconds between updates. The maximum time between TAP receiving a latitude update was 0.28 seconds, which is less than TAP's one second processing frame and was deemed acceptable. TAP received 2,725 UTC updates at a rate of one update per second, also deemed acceptable. The last row of Table 1 shows inter-arrival times of the complete active route from the FMC. The complete active route requires the receipt of multiple messages corresponding to each waypoint rather than a single message as was the case for present position longitude and UTC. The 16.28 seconds average complete active route inter-arrival time was acceptable since routes rarely change during the flight. A comparison of Stage 0 Hanger, Taxi, and Airborne tests (not shown) did not show much variation in parameter inter-arrival times. For this reason, only a few collections were required to achieve Stage 0 objectives.

Table 1. Sample inter-arrival times of TAP input parameters via STAP data feed from the UTAS AID ARINC 834 server during a Stage 0 airborne test conducted on an Alaska Airlines repositioning flight.

\begin{tabular}{l|c|c|c|c|}
\hline Input Parameter & $\begin{array}{c}\text { Updates } \\
\text { during Flight }\end{array}$ & $\begin{array}{c}\text { Average Inter-arrival } \\
\text { Time }(\mathrm{sec})\end{array}$ & $\begin{array}{c}\text { Maximum Inter- } \\
\text { arrival Time (sec) }\end{array}$ & $\begin{array}{c}\text { Standard Deviation of } \\
\text { Inter-arrival time (sec) }\end{array}$ \\
\hline Present Position Latitude & 12,910 & 0.21 & 0.28 & 0.02 \\
\hline UTC & 2,725 & 1.00 & 1.20 & 0.10 \\
\hline Complete Active Route & 150 & 16.28 & 60.29 & 9.93 \\
\hline
\end{tabular}

\section{Stage 1 Analysis and Results}

On revenue service flights of the three TAP-equipped aircraft, Alaska Airlines pilots launched a "TAP Utility" application installed on their iPads to collect Stage 1 in-flight data. ${ }^{16}$ This approach to deploy the utility application to all Alaska pilots, rather than just a select few, was taken to collect a wide range of destination airports and flight conditions for the Stage 1 analysis. The TAP Utility required minimal data entry by the pilots and launched TAP (without the TAP Display) using a "fire and forget" procedure such that TAP operated without pilot interaction or observation for the duration of the flight, just recording data for later analysis. TAP recorded data representing 26 unique Conterminous US (CONUS) airport destinations across 166 TAP instances. (A TAP instance is created every time a pilot launched TAP using the utility.) Pilots were asked, but not required, to launch the utility either at the gate or during flight. After launch, TAP recordings included: (1) the STAP data feed, (2) TAP messages used for postflight debugging, (3) ownship state, (4) ownship trajectory predictions, (5) TAP-generated reroutes and characteristics, and (6) data downloaded from the internet. All data was recorded on UTAS and GoGo hardware and transferred off manually, and later automatically via internet downloads, after multiple flights were completed.

During the first part of Stage 1, referred to as Stage 1a, in-flight internet connectivity was unavailable to TAP due to pending FAA approvals. TAP requires wind data to calculate candidate reroutes, which TAP receives via internet connectivity. If pilots launched the utility application on the ground, then TAP received internet data (including winds) via a terrestrial modem. If pilots launched the utility application in the air, then TAP did not receive internet data and no reroutes were calculated. Consequently, a separate process running at NASA recorded internet data to be used for post-flight analysis. During the second part of Stage 1, referred to as Stage 1b, internet connectivity was available throughout the flight to TAP. Of the 166 TAP instances collected, 126 were collected during Stage 1a and 40 were collected during Stage 1b. ${ }^{17}$

\section{Data Validation}

NASA and Engility validated Stage 1 data before performing quantitative analysis. There were three main validation steps. The first step confirmed that all expected TAP-generated data files were successfully recorded and transferred off the aircraft. This validation detected an issue, which was later resolved, recording data files downloaded from the internet. The second step confirmed that the flight was operated within the CONUS. Data collected on flights that started or ended outside the CONUS (e.g., Alaska, Hawaii, Mexico) were excluded from certain analyses since TAP's navigation database is currently limited to the CONUS. The third step confirmed that TAP ran all the way to

\footnotetext{
${ }^{16}$ Stage 1a in-flight testing began on September 26, 2017.
}

${ }^{17}$ Stage 1b in-flight testing began on January 1, 2018. 
the destination airport without stopping. There were instances where TAP stopped data collection soon after launch which were excluded from analysis. The likely cause was a utility application feature that allowed pilots to end data collection.

As discussed in Section II-C-1, a wiring design omission necessitated the acquisition of several air-data parameters from the FMC, rather than receiving them directly from the ADC; the TAP software deployed to the aircraft for Stage 1 was modified accordingly. Early analysis of Stage 1 data indicated that Mach number and altitude rate data received from the FMC were noisier than experienced during previous TASAR flight trials. Since Stage 1 did not have pilot inputs, the Stage 1 TAP software used state variables to identify and track inputs the pilot would have made signifying an update to the FMC active route (e.g., cruise Mach change). An active-route update was triggered when Mach changed by 0.01 (rounded to two decimal places). These active-route updates interrupted TAP's reroute generation since TAP was designed to discard existing reroutes and calculate new reroutes when the ownship active route was updated. As a result, the TAP software onboard produced infrequent reroutes during the Stage 1 flights.

For this reason, a post-flight "playback" methodology was employed whereby the STAP data recorded onboard was replayed on a Windows computer on the ground after the flight using a modified TAP software version. The TAP software was updated so that playbacks used ECON speed based on cost index to prevent frequent active-route updates. The noisy altitude rate also impacted TAP by occasionally triggering logic that caused TAP to predict an early descent to the destination airport. A TAP software change was made to handle altitude rate noise. This playback method provided TAP reroute advisories based on the data recorded in flight (though not on in-flight hardware) for use in Stage 1 data analysis, avoiding the cost and time delay of updating the TAP software running on Alaska Airlines aircraft during Stage 1.

\section{Data Analyses}

After the three-step data validation described above, the five analyses listed in Table 2 quantified TAP performance using either in-flight data directly or post-flight playback data as required. An " $x$ " in the "In-flight data" column indicates that TAP in-flight recorded data was analyzed. An " $x$ " in the "Post-flight playback data" column indicates that the analysis was based on TAP ownship predictions and reroute advisories generated by post-flight playbacks. These post-flight playbacks leveraged in-flight recording and ground recorded internet data to reflect TAP software changes that occurred after the start of Stage 1. The playback method was also used to identify and verify issues with actual flight data in an efficient manner, thereby enabling all software updates from Stage 1 to be consolidated into a single, high confidence, software deployment for Stage 2.

Table 2. Stage 1 analysis objectives.

\begin{tabular}{|c|l|c|c|}
\hline & Analysis Objective & $\begin{array}{c}\text { In-flight } \\
\text { data }\end{array}$ & $\begin{array}{c}\text { Post-flight } \\
\text { playback data }\end{array}$ \\
\hline 1 & Confirm TAP Received STAP Data & $\mathrm{x}$ & \\
\hline 2 & Confirm TAP Received Internet Data & $\mathrm{x}$ & \\
\hline 3 & Confirm UTAS/GoGo In-flight Hardware Performance & $\mathrm{x}$ & \\
\hline 4 & Demonstrate TAP Fuel and Time Accuracy is Acceptable & & $\mathrm{x}$ \\
\hline 5 & Verify TAP fuel and time outcomes are stable & $\mathrm{x}$ & $\mathrm{x}$ \\
\hline
\end{tabular}

Analysis 1: Confirm TAP Received STAP Data

The first row in Table 2 is the Stage 0 analysis described in the previous section (i.e., confirming that TAP received STAP data) applied to the dataset received in Stage 1 flights. The Stage 1 results did not significantly differ from Stage 0 , so statistics will not be presented.

Analysis 2: Confirm TAP Received Internet Data

For the second row in Table 2, post-flight analysis confirmed that TAP's internet download schedule for winds, weather hazards, and SUA activation schedule was achieved in-flight. Since the downloaded wind file size was much larger than the weather hazards and SUA schedule data file sizes, winds were the most likely data element to have a performance impact on internet data downloading. During 33 hours of Stage $1 \mathrm{~b}$ flight time, it took on average 53 seconds to download each wind file up to a maximum 187 seconds download time. This performance was considered acceptable, since winds are updated hourly. 
Analysis 3: Confirm UTAS/GoGo In-flight Hardware Performance

TAP-generated performance metrics were used to confirm TAP's performance on UTAS and GoGo hardware (third row in Table 2). TAP identifies and records if it cannot complete its scheduled reroute-advisory processing within its one-second processing cycle. This may indicate an issue if this condition occurs for an extended period of time. An evaluation of about 63 hours of TAP running on the AID during Stage 1a and about 9 hours on the ACPU during Stage $1 \mathrm{~b}$ showed a maximum of eleven consecutive seconds that this condition occurred which was acceptable.

\section{Analysis 4: Demonstrate TAP Fuel and Time Accuracy is Acceptable}

These first three analyses relied exclusively on data collected in-flight. The fourth row in Table 2, demonstrate TAP fuel and time accuracy is acceptable, leveraged post-flight TAP playbacks. The accuracy analysis compared TAP fuel and time predictions against the as-flown (i.e., actual) ownship trajectory. Alaska pre-departure flight plans, which contain fuel and time predictions at each waypoint along the flight plan, were also compared against the as-flown trajectory. First, fuel accuracy is discussed, then time accuracy.

The Fuel Factor Difference metric shown in Eq. (1) quantified TAP and Alaska pre-departure flight-plan fuel accuracy relative to as-flown trajectories. The metric was calculated between flight plan waypoints that were at least 30 minutes apart. Only predictions where the route or altitude did not change were analyzed since flight plan predictions were not updated after departure. The first term in Eq. (1) is the absolute value of the ratio of as-flown burn rate obtained from ownship state $(B R)$ to the flight plan predicted burn rate $\left(\widehat{B R_{F P}}\right)$ minus one. Burn rate is calculated as the aircraft weight difference between the two waypoints divided by the time difference. Ideally, the asflown burn rate $(B R)$ should equal the flight plan predicted burn rate $\left(\widehat{B R_{F P}}\right)$, so the minus one term quantifies the difference from the ideal burn rate. The second term in Eq. (1) similarly quantifies the absolute value of the difference between the ratio of the as-flown burn rate $(\mathrm{BR})$ to TAP's predicted burn rate $\left(\widehat{B R_{T A P}}\right)$ and one.

$$
\text { Fuel Factor Difference }=\left|\left(\frac{B R}{\widehat{B R_{F P}}}\right)-1\right|-\left|\left(\frac{B R}{B \widehat{B T A P}}\right)-1\right|
$$

The fuel factor difference metric results shown in Figure 8 are based on 11.7 hours of flight time. A mean of -0.01 shows that TAP's fuel prediction has a similar level of accuracy as the pre-departure flight plan. This similar accuracy increases confidence in TAP's fuel predictions. Positive values along the x-axis indicate that TAP had a better fuel prediction than the pre-departure flight plan and vice versa.

A similar analysis for time accuracy indicated that TAP's groundspeed prediction had a similar level of accuracy as the pre-departure flight plan relative to the as-flown trajectory (analysis results not shown). An additional analysis was performed in which TAP's groundspeed predictions relative to the FMC-predicted time to each waypoint were assessed. FMC predictions were updated post-departure and, therefore, a larger sample size was available to compare groundspeeds than was available for the analysis based on pre-departure flight-plan predictions. FMC fuel predictions were not available to TAP via the STAP data feed, so the fuel factor difference metric was not quantified for these predictions.

The groundspeed percent-error-difference metric in Eq. (2) was the method that quantified TAP and FMC groundspeed prediction accuracy. The first term in the equation is the absolute value of the percentage difference between FMC predictions between waypoints $\left(\widehat{G S_{F M C}}\right)$ and groundspeed calculated using ownship state position and time $(G S)$. The second term is similarly calculated as the absolute value of the percentage difference between TAP predictions $\left(\widehat{G S_{T A P}}\right)$ and groundspeed calculated from ownship state. The metric was calculated between active route waypoints that were at least 30 minutes apart in cases where the active route and cruise altitude

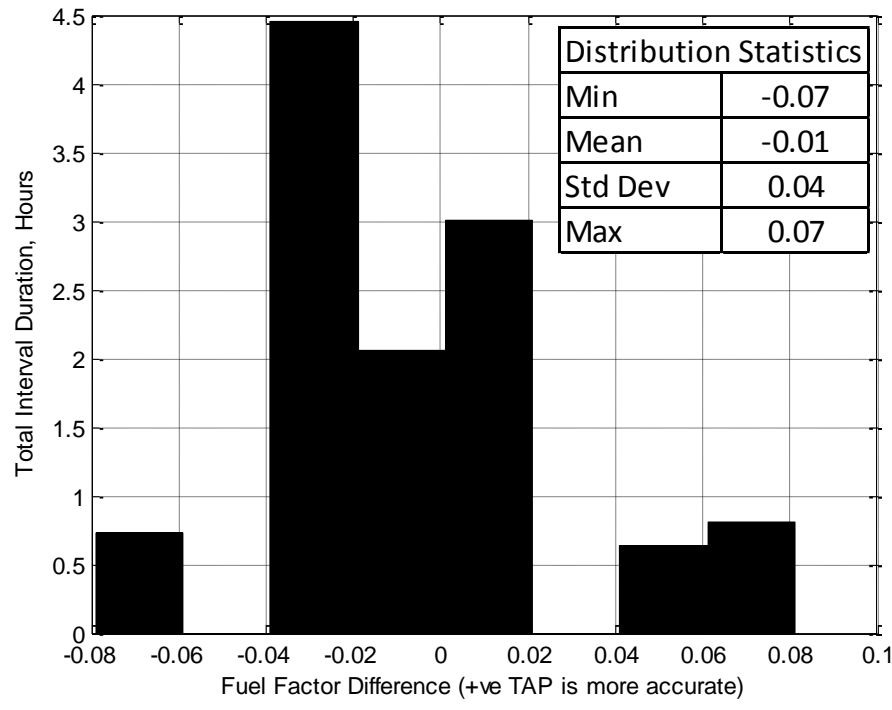

Figure 8. Distribution of fuel factor difference metric. Positive values indicate that TAP had a better fuel prediction than the pre-departure flight plan. Negative values indicate that the pre-departure flight plan had a better fuel prediction than TAP. 
did not change between those waypoints. The last FMC and TAP predictions prior to the first waypoint in the interval were used to calculate the metric.

$$
\text { Groundspeed Percent Error Difference }=\left|\left(\frac{G \widehat{S_{F M}}-G S}{G S}\right) 100 \%\right|-\left|\left(\frac{G \widehat{S_{T A P}}-G S}{G S}\right) 100 \%\right|
$$

Figure 9 shows the distribution of this error difference across about 54 hours of total interval duration. Positive values along the $\mathrm{x}$-axis indicate that TAP had better groundspeed predictions than the FMC and vice versa. The distribution mean of zero indicates that TAP and FMC had similar levels of groundspeed prediction accuracy relative to as-flown groundspeed. Similar FMC and TAP prediction accuracy increases confidence of the accuracy of TAP's advisories, particularly as it relates to predicted time savings.

Analysis 5: Verify TAP fuel and time outcomes are stable

The TAP fuel and time stability analysis listed on the last row of Table 2 identified large fuel or time outcome changes during Stage 1 that may indicate an accuracy issue and/or an issue that would negatively impact pilots' perception of the TAP software. One behavior that this analysis identified was due to fuel weight being received in $40 \mathrm{lbs}$ increments from the FMC. The ownship active route and advisory trajectory predictions were not synchronized which caused fuel outcomes to oscillate depending on whether a 40 lbs fuel change occurred between the ownship active route prediction and advisory trajectory prediction. The TAP software was later modified to synchronize these two predictions, which eliminated fuel outcome jumps due to the fuel weight increment. This discretization of fuel weight was not seen on other TASAR flight trial aircraft and thus is an example of how different aircraft have different data characteristics that must be considered when adapting TAP to new aircraft.

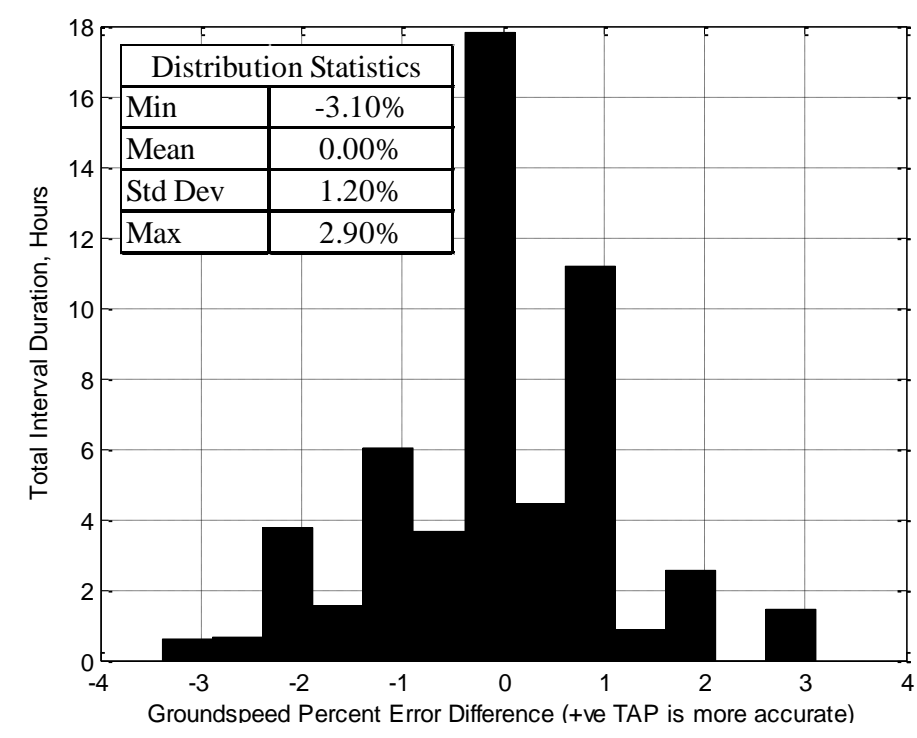

Figure 9. Distribution of groundspeed percent error metric. Positive values indicate TAP had a better groundspeed prediction than the FMC. Negative values indicate that the FMC had better groundspeed predictions than TAP.

The results of the Stage 0 and 1 analyses verified TAP's performance in acquiring data from the avionics and internet, operating on UTAS/Gogo hardware, and generating accurate and stable fuel and time predictions. Software bugs identified during the analyses were fixed and verified using playbacks of recorded STAP data, and the updated software has been delivered for installation in the aircraft. These analyses indicate readiness to proceed with Stage 2 of the operational evaluation, where pilots will evaluate TAP in revenue service operations.

\section{Conclusion}

Bringing Alaska Airlines to a state of operational readiness for evaluating NASA's concept and technology for Traffic Aware Strategic Aircrew Requests (TASAR) in revenue service, the process for which was described in detail in this paper, has been a complex but successful multidisciplinary endeavor between NASA, its contractors, Alaska Airlines, and its industry partners. Complex endeavors take time to accomplish, and this collaborative government/airline/industry effort was no exception. Following the kickoff meeting in September 2015, the first onaircraft ground testing occurred 18 months later in March 2017, followed in another six months in September 2017 by the first commercial airline flight with NASA's TASAR software, Traffic Aware Planner (TAP), operating onboard (in a test mode). After about another six months, the TASAR operational evaluation will begin with pilots using TAP to compute route changes compatible with nearby traffic and airspace constraints to improve flight efficiency.

Achieving TASAR operational readiness at Alaska Airlines involved a host of interdependent activities: hardware/software installations and modifications, establishment of Electronic Flight Bag connectivity to avionics and airborne internet, FAA certifications and operational approvals, adapting TAP to the specific aircraft performance and avionics environment, the preparation of pilot training materials, and an extensive amount of coordinated system 
testing. The multi-stage implementation plan for testing proved to be a cost-effective and efficient approach for verifying the system design and for identifying and resolving issues prior to commencing the operational evaluation with pilots.

With operational readiness now achieved, the TASAR evaluation by Alaska Airlines and NASA will take place on a daily basis over a number of months as Alaska's three TAP-equipped B737-900ER aircraft and multiple TASARtrained pilots cross the country transporting passengers to their destinations while seeking more cost-effective routes to do so. The evaluation will gather feedback from line pilots, assess achieved benefits of time and fuel savings, and determine what adjustments in functionality, procedures, and training are needed to achieve satisfactory performance for airline operations of TASAR.

Meanwhile, Alaska and NASA have already planned the next phase of the collaboration leveraging NASA air and ground technologies to enhance coordination between pilots and dispatchers for airborne reroutes. By integrating NASA's air and ground rerouting technologies, additional benefits are anticipated for both individual flights and fleet management, while further increasing the likelihood of ATC approval of reroute requests.

\section{References}

[1] M. G. Ballin and D. J. Wing, "Traffic Aware Strategic Aircrew Requests," in AIAA-2012-5623, 12th AIAA Aviation Technology, Integration, and Operations Conference, Indianapolis, 2012.

[2] Gogo, From the Ground Up: How the Internet of Things will Give Rise to Connected Aviation, Chicago: Gogo, LLC, 2016.

[3] S. E. Woods, R. A. Vivona, J. Henderson, D. J. Wing and K. E. Burke, "Traffic Aware Planner for Cockpitbased Trajectory Optimization," in AIAA-2016-4067, 16th AIAA Aviation Technology, Integration, and Operations Conference, Washington DC, 2016.

[4] D. J. Wing, M. G. Ballin, S. Koczo and R. A. Vivona, "Developing an On-Board Traffic-Aware Flight Optimization Capability for Near-Term Low-Cost Implementation," in AIAA-2013-4231, 13th AIAA Aviation Technology, Integration, and Operations Conference, Los Angeles, 2013.

[5] D. J. Wing, "Achieving TASAR Operational Readiness," in AIAA-2015-3400, 15th AIAA Aviation Technology, Integration, and Operations Conference, Dallas, 2015.

[6] J. Henderson, H. Idris and D. J. Wing, "Preliminary Benefits Assessment of Traffic Aware Strategic Aircrew Requests (TASAR)," in AIAA-2012-5684, 12th AIAA Aircraft Technoogy, Integration, and Operations Conference, Indianapolis, 2012.

[7] J. Henderson, "Annualized TASAR Benefit Estimate for Virgin American Operations," NASA/CR-2015218786, 2015.

[8] J. Henderson, "Annualized TASAR Benefit Estimate for Alaska Airlines Operations," NASA/CR-2015-218787, 2015.

[9] S. Koczo, "Analysis of Operational Hazards and Safety Requirements for Traffic Aware Strategic Aircrew Requests (TASAR)," NASA/CR-2013-218002, 2013.

[10] S. Koczo, "TASAR Certification and Operational Approval Requirements - Analyses and Results," NASA/CR2015-218708, 2015.

[11] D. A. Roscoe, R. A. Vivona, S. E. Woods, D. A. Karr and D. J. Wing, "Deploying a Route Optimization EFB Application for Commercial Airline Operational Trials," in Digital Avionics Systems Conference, Sacramento, CA, 2016.

[12] J. M. Maris, M. A. Haynes, D. J. Wing, K. A. Burke, J. Henderson and S. E. Woods, "Traffic Aware Planner (TAP) Flight Evaluation," in AIAA-2014-2166, 14th AIAA Aircraft Technology, Integration, and Operations Conference, Atlanta, 2014.

[13] D. A. Karr, R. A. Vivona, S. E. Woods and D. J. Wing, "Point-Mass Aircraft Trajectory Prediction Using a Hierarchical, Highly-Adaptable Software Design," in AIAA Modeling and Simulation Technologies Conference, Denver, CO, 2017. 
[14] International Air Transport Association, "Standardised Computerised Aircraft Performance (SCAP) Task Force," 2018. [Online]. Available: http://www.iata.org/whatwedo/workgroups/Pages/scaptf.aspx. [Accessed April 2018].

[15] Eurocontrol, "Base of Aircraft Data (BADA)," 2018. [Online]. Available: http://www.eurocontrol.int/services/bada. [Accessed 24 April 2018]. 